Original article

Paediatrics Today 2015;11(2):160-171

DOI $10.5457 / \mathrm{p} 2005-114.122$

\title{
HEALTH-RELATED QUALITY OF LIFE AND PSYCHOLOGICAL DISTRESS IN ITALIAN PATIENTS WITH CYSTIC FIBROSIS
}

\author{
Vincenzo DI CIOMMO ${ }^{1 *}$, Eva MAZZOTTI ${ }^{1}$, Vincenzina LUCIDI $^{2}$, Simone PIGA ${ }^{1}$, Sergio BELLA ${ }^{2}$, \\ Marina CUTTINI ${ }^{1}$
}

${ }^{1}$ Unit of Epidemiology, Medical Directorate Bambino Gesù Children's Hospital-IRCCS Rome, Italy, ${ }^{2}$ Cystic Fibrosis Unit Department of Paediatrics, Bambino Gesù Children's Hospital-IRCCS , Rome, Italy

\footnotetext{
*Corresponding author: vincenzo.diciommo@gmail.com Tel.: + 39063612797

Fax.: + 390668592853
}

Received: July 29, 2014

Accepted: September 1, 2015

Key words: Children - Adolescents - Adults - Cystic fibrosis • Psychological distress.
Objective - The aim of this paper is to describe the health related quality of life (HRQoL) in an unselected hospital-based cohort of patients with Cystic fibrosis (CF), and to explore the association between HRQoL and psychological status. Study design - 115 patients with CF (47 children, 26 adolescents and 42 adults) attending the Bambino Gesù Pediatric Hospital in Rome, Italy, were recruited. HRQoL was measured through the Cystic Fibrosis Questionnaire (CFQ), child and adult versions. The Youth Self Report Form and the General Health Questionnaire were used to screen for psychological distress in patients aged below and above 18 respectively. Multivariable logistic regression analysis was performed to explore the relationship between CFQ scores and psychological distress, adjusting for socio-demographic and clinical variables. Results - HRQoL was high, except for Social Limitations, Energy/Wellbeing and Treatment Constraints. Twenty-eight patients $(24.4 \%)$ scored positive for psychological distress. HRQoL scores below median for Symptoms, Body Image and Social Limitations, and unemployment were significantly associated with the presence of psychological distress, while age, screening-based diagnosis, gender and medical factors were not. Conclusions - Screening for psychological distress should be part of routine management of patients with CF. Opportunities for age-appropriate socialization, work and leisure time should be allowed and encouraged.

\section{Introduction}

Cystic fibrosis (CF) is a recessively inherited genetic disease, characterized by a range of clinical manifestations, including chronic airway infection (1). The disease progresses through childhood into young adulthood, with death usually occurring as a result of chronic pulmonary disease. Better patient management and medical care have greatly improved life expectancy over the last few decades (1), but the burden of treatment remains significant. As a consequence, the focus of the attention of clinicians and researchers has moved from mere survival to the assessment of patients' wellbeing and psychosocial conditions. Health-related quality of life (HRQoL), defined as the patients' "subjective evaluation of the influences of their current health status on the ability to achieve and maintain a level of overall functioning" (2) is increasingly used as an outcome measure in follow-up studies and after lung transplantation, as the end-point of interest in randomized controlled trials, and an 
indicator of appropriate management by CF centres.

So far, most studies of HRQoL in patients with CF have considered adults $(3,4,5)$ or adults and adolescents 6 separately from children (7). Disease severity showed an impact on HRQoL, together with gender in adults and adolescents (females had poorer HRQoL (6). Children were surveyed to assess the effect of newborn screening, which was not found associated with different HRQoL (7). More recently an investigation in Hungarian patients from paediatric age to young adulthood has been published, revealing hospitalisation, infection from Pseudomonas and malnutrition as significant, negative predictors of a low HRQoL (8). We hypothesized that patients in different stages of their life have different forms of adaptation to CF, and psychological distress, amenable to useful interventions, can be associated with HRQoL.

The aim of this paper, using a non-selected hospital-based series of patients with CF, was to explore the HRQoL across a broader lifespan, ranging from childhood to adulthood, and to assess psychological distress and its relationship with HRQoL.

\section{Methods}

\section{Participants}

The study was carried out in 2005 at the CF Unit of the Bambino Gesù Children's Hospital, a tertiary referral hospital in Rome, Italy. We considered all patients with documented CF (positive sweat test, followed by genetic testing) regularly attending the Unit. Additional inclusion criteria were age 7 years or more and the ability to read Italian. Patients were consecutively enrolled and asked to complete the study questionnaires during a routine hospital visit or admission, not associated with exacerbation or acute illness. Written informed consent was obtained from the patients, or from the parents if the pa- tient was less than 18 years old. Prior to data collection, ethical and scientific approval was granted by the hospital board for clinical research. Out of 121 eligible patients, 118 agreed to participate (response rate 97.5\%). Three patients ( 1 child, 1 adolescent and 1 adult) were excluded because of missing values in the psychological distress questionnaires. Thus, the analyses for this paper were carried out on 115 patients.

\section{Instruments and data collection}

A structured form was used to record information on the patients' age, gender, and socio-demographic information. Data abstracted from clinical records included age and type of CF diagnosis (i.e. neonatal screening or based on clinical signs and symptoms); the number of exacerbations of respiratory symptoms during the previous year (defined according to Fuchs (9) as treatment with parenteral antibiotics for any 4 of a list of 12 symptoms); and the condition at the time of the study (height and weight, presence of chronic respiratory infection, and of comorbidities, such as impaired glucose intolerance, diabetes and liver problems). The most recent (i.e. within one month before the interview) results of the pulmonary function test (percentage of predicted Forced Expiratory Volume at one second, or FEV1\%) and chest Xray scores were recorded. Standardized selfcompleted questionnaires mentioned below were used for the assessment of quality of life and psychological conditions.

The Cystic Fibrosis Questionnaire (CFQ) is a validated disease-specific instrument for measuring HRQoL in patients with CF (10, 11). Different versions are available for children aged 7 to 13 years (CFQ-child), and for adolescents and adults $\geq 14$ (CFQ-teen/adult) (11). The adult version includes 47 items grouped in 13 domains or subscales: Physical Functioning, Role, Energy and Wellbeing, Emotions/interrelations, Social Limitations, 
Embarrassment, Body Image, Eating Disturbances, Treatment Burden, Respiratory and Digestive Symptoms, and Health Perception. The CFQ-child is similar, but includes only 33 items for nine domains, since those related to Role, Embarrassment, and Health perceptions are missing. Both the adult and the child versions take about 20 minutes to complete. Within each domain, scores are computed by summing up the coded answers to the relevant items. Higher scores indicate better quality of life. An example of one of the items of Emotional Functioning is "You felt sad and depressed". For this study, the child and the teen/adult CFQ French versions were translated into Italian and backtranslation into the original language was carried out to assess the validity of the translation. Participants were asked to complete the questionnaires with reference to the two previous weeks, as suggested in the original questionnaires (11).

Psychological status was assessed using two different instruments, according to the age of the respondent. For the adult patients (18 years or older) we used the 12-item General Health Questionnaire (GHQ-12) (12). This is a widely used self-completed questionnaire, designed to screen for acute psychological distress and non-psychotic depressive and/or anxiety psychiatric disorders. The scale asks whether the respondent has experienced a given symptom or behaviour recently. An example is: "Have you felt useful?" Each item is rated on a 4 point Likerttype scale (from less than usual to much more than usual). The reliability and validity of the Italian version have already been documented (13).

The Youth Self Report Form (YSR) was used for children and adolescents (14). This is a self-completed questionnaire developed to measure emotional and behavioural problems in patients up to 18 years of age. It includes 112 items describing a wide range of emotional and behavioural problems experienced over the past six months, to be answered on a three point scale from 0 ("not true") to 2 ("very true" or "very often true"). Measurements consistent with the diagnostic categories of the American Psychiatric Association Diagnostic and Statistical Manual of Mental Disorders (Affective, Anxiety, Somatic, Attention/Hyperactivity, Opposition$\mathrm{al}$ and Conduct problems) are derived from the scores (14). For this paper the Somatic oriented scale was excluded from the analysis (15) to avoid auto-correlation with the domain of Symptoms of CFQ questionnaire.

\section{Data analysis}

CFQ scores were standardized to a 0-100 points scale ( $\mathrm{T}$ values), to allow comparability of scores across the different domains (12). Standardised $T$ values ranging from 0 to 100 (mean 50, standard deviation 10). Missing values ( $<20 \%$ in each domain) were replaced by the patient's mean value on the other items of the same domain. Gastrointestinal and respiratory symptoms were considered together for this analysis. The GHQ-12 was scored using the binary method (0-1) (14). To receive a score of 1 for a given item a respondent should answer that he or she experienced a specific problem (e.g. difficulty concentrating) "more than usual" or "much more than usual". Thus, the resulting total score is bound to vary between 0 and 12 . Patients scoring 4 or more are operationally defined as "cases", i.e. they score positive for acute depressive and/or anxiety disorder. For YSR, the 93th centile of the normative population was used to separate normal from borderline and pathological scores (14). In other words, "cases" were those who tested positive (above the aforementioned cut-off scores) as patients at risk for psychological distress, to be fully evaluated, while "non-cases" were patients who could be discarded from further 
evaluation. We computed combined scores to allow comparison across the different patient age groups. For the combined CFQ scores, only domains common to both the child and teen/adult versions of the instrument were used. A combined psychological distress category included as "cases" patients with GHQ-12 scores $\geq 4$ for adults, and those scoring equal or above the 93th centile of the normative population in at least one YSRDSM oriented scale for children and adolescents (Somatic scale excluded).

\section{Ethics statement}

The study was approved by the Institutional Research Board. The adult patients signed an informed consent, while for patients $<18$ years-old the consent was signed by a parent (the study was always discussed with the patient if his/her age was $>14$ years.)

\section{Statistical analyses}

Data are presented as proportions or medians and interquartile ranges (IRQ) for categorical and continuous variables respectively. Differences between proportions were tested with the Chi-squared or the Fisher exact test, as appropriate. The non-parametric WilcoxonMann-Whitney test and the Kruskal-Wallis analysis of variance were used in the case of continuous variables. A multivariable logistic regression model was used to assess the relationship between quality of life scores and psychological distress (i.e. being a "case"), adjusted for socio-demographic and medical factors. Quality of life levels were categorized as CFQ specific domain scores either below or above the median. The Emotional Status items were excluded for this analysis. Additional variables considered for inclusion in the model were: the patient's sex and age; parental education (below or above 8 years of school); and the patient's occupational status, defined as being a student, employed, or un- employed. The following medical factors were considered: FEV1\%; presence of chronic airway infection; colonization by Pseudomonas aeruginosa; number of exacerbations over the previous 12 months; BMI; and short stature, defined as height below the $10^{\text {th }}$ centile of the Italian growth charts (16). Age at CF diagnosis and type of diagnosis (i.e. based on signs and symptoms versus screening) were also taken into account as possible predictors. The final model retained all the variables significantly associated with the presence of psychological distress at a $\mathrm{p}<0.10$ level, plus the patient's age and sex. To be at risk means the occurrence of the outcome more often in patients exposed to the variable than in those not exposed, while patients said to be protected refers to the opposite, i.e. the patients with a variable incur it less frequently than those without. Statistical analysis was carried out using the STATA package, version 10.0 (StataCorp 2007.Stata Statistical software: Release 10. College Station,Tx: StataCorp).

\section{Results}

\section{Patient characteristics}

The study population included 47 children aged $7-13$ years old (40.9\%), 26 adolescents between 14 and 17 years (22.6\%), and 42 young adults 18 year or more $(36.5 \%)$. Fiftyseven $(49.6 \%)$ were males (Table 1$)$.

Most of them were students $(72.2 \%$, ranging from $100 \%$ in children to $38.1 \%$ in adults). Among the adults out of school, 11 $(42.3 \%)$ were unemployed.

The median patient age at diagnosis decreased from 5 years among the adults to less that 1 year in children, documenting a significant advancement with year of birth $(\mathrm{p}<0.001)$. Only about $15 \%$ of patients ( $23.4 \%$ of children, $4.8 \%$ of adults, $\mathrm{p}=0.041$ ) were diagnosed by screening.

At the time of the study, the overall median value of FEV1\% was 84 (IQR 70-97); 62.6\% 


\begin{tabular}{|c|c|c|c|c|}
\hline Variables & $\begin{array}{l}\text { Sample } \\
(\mathrm{n}=115)\end{array}$ & $\begin{array}{l}\text { Children* } \\
(\mathrm{n}=47)\end{array}$ & $\begin{array}{l}\text { Adolescents } \dagger \\
(\mathrm{n}=26)\end{array}$ & $\begin{array}{l}\text { Adults } \neq \\
(\mathrm{n}=42)\end{array}$ \\
\hline \multicolumn{5}{|l|}{ Demographic variables } \\
\hline Males, n (\%) & 57 (49.6) & $23(48.9)$ & $15(57.7)$ & $19(45.2)$ \\
\hline Lazio residents, n (\%) & $81(70.4)$ & $32(68.1)$ & $16(61.5)$ & $33(78.6)$ \\
\hline \multicolumn{5}{|l|}{ Occupational status, n (\%) } \\
\hline Student & $83(72.2)$ & $47(100.0)$ & $20(76.9)$ & $16(38.1)$ \\
\hline Employed & $19(16.5)$ & - & $4(15.4)$ & $15(35.7)$ \\
\hline Unemployed & $13(11.3)$ & - & $2(7.7)$ & $11(26.2)$ \\
\hline Maternal education $>8$ yrs, $\mathrm{n}(\%)$ & $59(52.2)$ & $27(58.7)$ & $12(48.0)$ & $20(47.6)$ \\
\hline Paternal education $>8$ yrs, $\mathrm{n}(\%)$ & $66(57.4)$ & $28(59.6)$ & $16(61.5)$ & $22(52.4)$ \\
\hline \multicolumn{5}{|l|}{ Clinical variables } \\
\hline Age at diagnosis, yrs, median, IQR & $1(0-5)$ & $0(0-1)$ & $1(0-8)$ & $5(0-10)$ \\
\hline Signs-symptoms & $98(85.2)$ & $36(76.6)$ & $22(84.6)$ & $40(95.2)$ \\
\hline Screening & $17(14.8)$ & $11(23.4)$ & $4(14.4)$ & $2(4.8)$ \\
\hline FEV1 \%, median, IQR & $84(70-97)$ & $91(79-100)$ & $92(74-100)$ & $72(46-84)$ \\
\hline $\mathrm{N}$ of PE in last 12 mos, median, IQR & $1(0-2)$ & $0(0-1)$ & $1(0-2)$ & $1(0-2)$ \\
\hline Presence of chronic lung infection, $\mathrm{n}(\%)$ & $72(62.6)$ & $19(40.4)$ & $17(65.4)$ & $36(85.7)$ \\
\hline Colonization by PA, n (\%) & $58(50.4)$ & $16(34.0)$ & $14(53.9)$ & $28(66.7)$ \\
\hline Presence of comorbidity, n (\%) & $43(37.4)$ & $14(29.8)$ & $3(11.5)$ & $26(61.9)$ \\
\hline BMI, median, IQR & $20(18-23)$ & $18(16-20)$ & $21(19-24)$ & $21(18-23)$ \\
\hline Height $>10^{\text {th }}$ centile, $\mathrm{n}(\%)$ & $41(35.7)$ & $20(42.6)$ & $12(46.2)$ & $9(21.4)$ \\
\hline
\end{tabular}

$\mathrm{IQR}=$ Interquartile range; $\mathrm{FEV}=$ Forced expiratory volume; $\mathrm{N}=$ Number; $\mathrm{PE}=\mathrm{Pulmonary}$ exacerbations $\mathrm{PA}=$ Pseudomonas aeruginosa BMI=Body mass index; ${ }^{\top} 7-13$ yrs; ${ }^{\dagger} 14-17$ yrs; ${ }^{\ddagger} \geq 18$ yrs.

of patients had chronic airways infection, and 50.4\% Pseudomonas aeruginosa colonization; in $37.4 \%$ comorbidities were present (osteoporosis in 15 , liver disease in 12 , diabetes in 12 and 5 gastro-oesophageal reflux). All these clinical characteristics significantly worsened with increasing patient age $(\mathrm{p}<0.05)$.

Table 2 shows the results of quality of life measurement (CFQ scores) and the proportion of patients scoring positive for psychological distress by age group. The overall median values of CFQ were $\geq 80$ in all domains, except Social Limitations, Energy/Wellbeing, and Treatment Burden. Median scores for Social Limitations and Eating Disturbances were significantly lower in children than in older patients, while the opposite was true for Energy/Wellbeing.
Almost one fourth of patients $(28 \%$, or $24.4 \%)$ scored positive for psychological distress (i.e. they were defined as "cases"), and the proportion increased with age, although not significantly (Table 2). Compared to "non-cases", "cases" had significantly lower HRQoL scores in most domains, except Social Limitations, Treatment Burden and Eating Disturbances (Fig. 1).

Table 3 shows the univariate association between psychological distress and sociodemographic and clinical variables. "Cases" were significantly more frequent among the unemployed patients and. No significant relationships with other clinical indicators (BMI, infections, exacerbations and comorbidities) were found. The proportion of psychological distress was significantly higher in 


\begin{tabular}{|c|c|c|c|c|c|}
\hline Scales & $\begin{array}{l}\text { Sample } \\
(\mathrm{n}=115)\end{array}$ & $\begin{array}{l}\text { Children* } \\
(\mathrm{n}=47)\end{array}$ & $\begin{array}{l}\text { Adolescents } \dagger \\
(\mathrm{n}=26)\end{array}$ & $\begin{array}{l}\text { Adults } \neq \\
(\mathrm{n}=42)\end{array}$ & $\mathrm{p}^{\S}$ \\
\hline \multicolumn{6}{|l|}{ CFQ scores (median; IQR) } \\
\hline Physical functioning & $89(75-96)$ & $94(83-100)$ & $91(71-96)$ & $85(75-96)$ & 0.238 \\
\hline Emotional state & $80(67-92)$ & $83(75-92)$ & $80(67-93)$ & $80(67-87)$ & 0.296 \\
\hline Social limitations & $67(44-83)$ & $44(33-56)$ & $75(67-83)$ & $79(67-92)$ & $<0.001$ \\
\hline Energy, wellbeing & $67(58-83)$ & $83(67-92)$ & $67(58-83)$ & $67(50-75)$ & $<0.001$ \\
\hline Body image & $89(67-100)$ & $89(78-100)$ & $78(56-89)$ & $89(67-100)$ & 0.074 \\
\hline Treatment constraints & $67(50-67)$ & $67(33-83)$ & $67(50-67)$ & $50(50-67)$ & 0.857 \\
\hline Eating disturbances & $89(67-100)$ & 78 56-100) & $100(83-100)$ & $100(67-100)$ & 0.042 \\
\hline Symptoms" & $80(73-87)$ & $80(67-87)$ & $81(77-85)$ & $81(65-85)$ & 0.785 \\
\hline \multicolumn{6}{|c|}{ Psychological distress (n; \%) } \\
\hline Cases** $^{* *}$ & $28(24.4)$ & $8(17.0)$ & 7 (26.9) & $13(31.0)$ & $0.293^{\dagger \dagger}$ \\
\hline
\end{tabular}

"7-13 yrs; ${ }^{\dagger} 14-17$ yrs; ${ }^{\ddagger} \geq 18$ yrs; ${ }^{\circledR}$ Kruskal-Wallis test; "Respiratory and digestive symptoms; ${ }^{* *} \mathrm{GHQ}-12$ score $\geq 4$ or score in at least one YSR-DSM oriented scale $\geq 93^{\circ}$ of normative population; ${ }^{\dagger \dagger}$ Chi-square test.

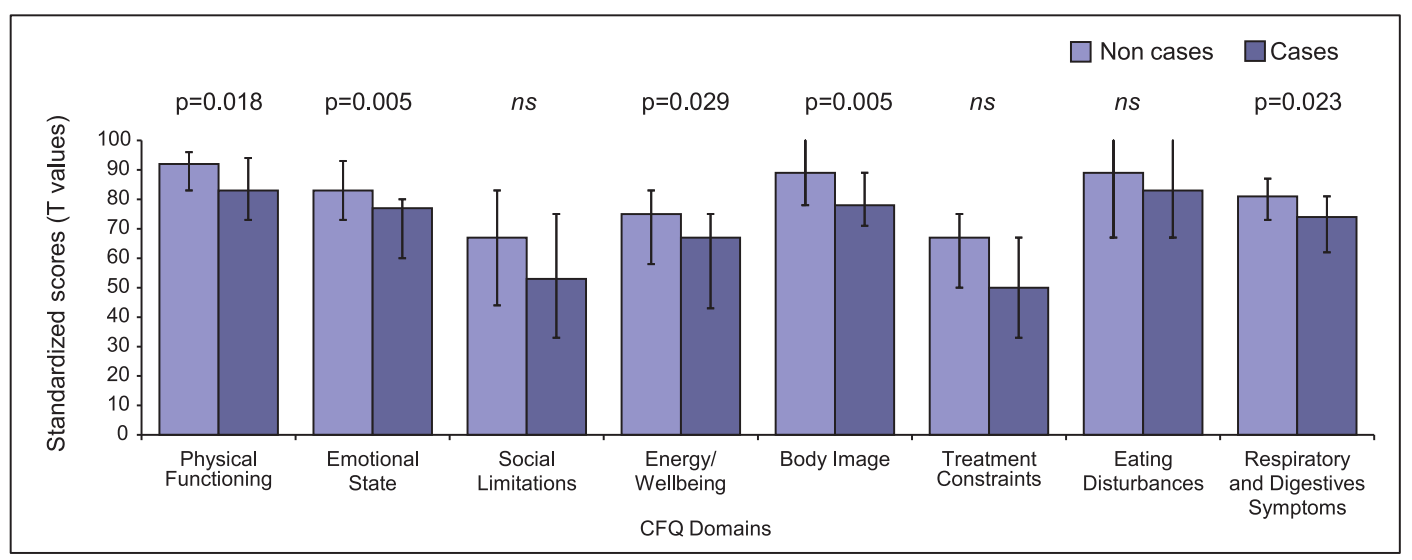

$\mathrm{CFQ}=$ Cystic Fibrosis Questionnaire; *Wilcoxon rank-sum (Mann-Whitney) test.

Fig. 1 Quality of life (CFQ scores, median and IQR) by presence of psychological distress.

patients diagnosed by signs and symptoms $(27.6 \%)$ than by screening (5.9\%) ( $\mathrm{p}=0.04)$.

The results of the multivariable analysis exploring the relationship between HRQoL measurements (scores below or above the median subscale value) and the presence of psychological distress are shown in Table 4.

Only factors significantly associated with the outcome of interest at the level of $\mathrm{p}<0.10$, plus age and sex, were retained in the final model. A HRQoL score below the median in Social Limitations, Body Image and Symptoms was associated with a four-fold to six-fold statistically significant increase in the likelihood of being screened positive for psychological distress testing. Being unemployed was the strongest risk factor, with an odds ratio as high as 8.31 ( $\mathrm{p}=0.02$ ) after adjustment for patient age and other covariates. The protective effect of a screening-based CF diagnosis found in univariate analysis was confirmed (OR 0.12; 95\% CI 0.01-1.09), although the statistical significance was marginal $(\mathrm{p}=0.06)$. Neither gender nor clinical variables showed any effect. 
Table 3 Prevalence of psychological distress a by demographic and clinical variables

\begin{tabular}{|c|c|c|c|}
\hline \multirow{3}{*}{ Variabiles } & \multicolumn{2}{|c|}{ Presence of psychological distress } & \multirow{3}{*}{$\mathrm{p}$} \\
\hline & No & Yes (cases) & \\
\hline & $(\mathrm{n}=87)$ & $(\mathrm{n}=28)$ & \\
\hline \multicolumn{4}{|l|}{ Demographic variables } \\
\hline \multicolumn{4}{|l|}{ Lazio residents, n (\%) } \\
\hline Yes & $61(75.3)$ & $20(24.7)$ & $0.895^{c}$ \\
\hline No & $26(76.5)$ & $8(23.5)$ & \\
\hline \multicolumn{4}{|l|}{ Gender, n (\%) } \\
\hline Male & $42(73.7)$ & $15(26.3)$ & $0.276^{c}$ \\
\hline Female & $45(77.6)$ & $13(22.4)$ & \\
\hline \multicolumn{4}{|l|}{ Occupational status, n (\%) } \\
\hline Student & $69(83.1)$ & $14(16.9)$ & $<0.050^{c}$ \\
\hline Employed & $12(63.2)$ & $7(36.8)$ & \\
\hline Unemployed & $6(46.2)$ & $7(53.9)$ & \\
\hline \multicolumn{4}{|l|}{ Maternal education, $\mathrm{n}(\%)$} \\
\hline$>8$ yrs & $49(83.0)$ & $10(16.9)$ & $0.070^{c}$ \\
\hline$\leq 8$ yrs & $37(68.5)$ & $17(31.5)$ & \\
\hline \multicolumn{4}{|l|}{ Paternal education, n (\%) } \\
\hline$>8 y r s$ & $51(77.3)$ & $15(22.7)$ & $0.638^{c}$ \\
\hline$\leq 8 y r s$ & $36(73.5)$ & $13(26.5)$ & \\
\hline \multicolumn{4}{|l|}{ Clinical variables } \\
\hline FEV1 \%, median, IQR & $87(70-99)$ & $77(56-91)$ & $0.058^{b}$ \\
\hline Age at diagnosis (yrs) median, IQR & $0(0-5)$ & $2(0-8)$ & $0.063^{\mathrm{b}}$ \\
\hline \multicolumn{4}{|l|}{ Type of diagnosis, n (\%) } \\
\hline Signs/symptoms & $71(72.5)$ & $27(27.6)$ & $0.040^{c}$ \\
\hline Screening & $16(94.2)$ & $1(5.9)$ & \\
\hline FEV1 \%, median, IQR & $87(70-99)$ & $77(56-91)$ & $0.058^{\mathrm{b}}$ \\
\hline $\mathrm{N}$ of PE in last 12 mos., median IQR & $1(0-2)$ & $0.5(0-1.5)$ & $0.769^{\mathrm{b}}$ \\
\hline \multicolumn{4}{|l|}{ Chronic lung infection, $\mathrm{n}(\%)$} \\
\hline Yes & $52(72.2)$ & $20(27.8)$ & $0.267^{c}$ \\
\hline No & $35(81.4)$ & 8 (18.6) & \\
\hline \multicolumn{4}{|l|}{ Colonization by PA, n (\%) } \\
\hline Yes & $47(82.5)$ & $10(17.5)$ & $0.092^{\mathrm{c}}$ \\
\hline No & $47(82.5)$ & $10(17.5)$ & \\
\hline \multicolumn{4}{|l|}{ Comorbidity, n (\%) } \\
\hline Yes & $29(67.4)$ & $14(32.6)$ & $0.113^{\mathrm{c}}$ \\
\hline No & $58(80.6)$ & $14(19.4)$ & \\
\hline BMI, median, IQR & $20(17-23)$ & $20(18-22)$ & $0.911^{\mathrm{b}}$ \\
\hline \multicolumn{4}{|l|}{ Height, n (\%) } \\
\hline$\geq 10$ centile & $58(78.4)$ & $16(21.6)$ & $0.360^{c}$ \\
\hline$<10$ centile & $29(70.7)$ & $12(29.3)$ & \\
\hline
\end{tabular}

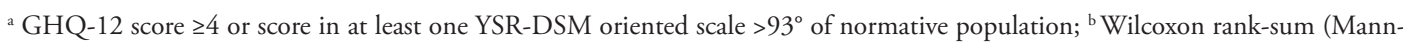
Whitney) test; Chi square or Fisher exact test; IQR=Interquartile range; $\mathrm{N}=$ Number; PE=Pulmonary exacerbations; PA=Pseudomonas aeruginosa.

When we restricted the analysis, only taking into consideration the children aged 1113 years, at whom the CBCL-YSR is aimed, the total sample was reduced to 94 patients, 26 aged 11-13 years; no major difference was observed in this sub-analysis, where no difference was found in the domain of Energy/ wellbeing and no difference was observed across the age groups of the domain Eating Disturbances. 


\section{Table 4 Factors associated with presence of psychological distress (logistic analysis)}

\begin{tabular}{|c|c|c|c|}
\hline \multirow{2}{*}{ Factors } & \multicolumn{2}{|c|}{ Psychological distress } & \multirow{2}{*}{$\mathrm{p}$} \\
\hline & Adjusted OR & $95 \% \mathrm{CI}$ & \\
\hline Age (yrs) & 1.09 & $0.98-1.22$ & 0.12 \\
\hline \multicolumn{4}{|l|}{ Gender } \\
\hline Males & 1.00 & & \\
\hline Females & 0.71 & $0.24-2.08$ & 0.53 \\
\hline \multicolumn{4}{|l|}{ Occupational status } \\
\hline Student & 1.00 & & \\
\hline Employed & 1.85 & $0.31-11.14$ & 0.50 \\
\hline Unemployed & 8.31 & $1.35-51.23$ & 0.02 \\
\hline \multicolumn{4}{|l|}{ Type of diagnosis } \\
\hline Signs/symptoms & 1.00 & & \\
\hline Screening & 0.12 & 0.01-1.09 & 0.06 \\
\hline \multicolumn{4}{|c|}{ CFQ social limitations below median } \\
\hline No & 1.00 & & \\
\hline Yes & 4.26 & $1.28-14.14$ & 0.02 \\
\hline \multicolumn{4}{|c|}{ CFQ body image below median } \\
\hline No & 1.00 & & \\
\hline Yes & 6.44 & $1.64-25.26$ & 0.01 \\
\hline \multicolumn{4}{|l|}{ CFQ symptoms } \\
\hline No & 1.00 & & \\
\hline Yes & 5.08 & $1.60-16.15$ & 0.01 \\
\hline
\end{tabular}

$\mathrm{OR}=\mathrm{Odds}$ ratio; $\mathrm{CFQ}=$ Cystic Fibrosis Questionnaire.

The proportion of psychological distress was not significantly higher in patients diagnosed by signs and symptoms $(31.3 \%)$ than by screening $(7.1 \%)(\mathrm{p}=0.10)$. The protective effect of screening-based CF diagnosis found in univariate analysis was confirmed, but no statistical significance could be shown (OR 0.09; 95\% CI 0.01-0.90, ( $\mathrm{p}=0.04)$, while Social Limitation, low Body image and Symptoms were confirmed as significant risk factors for psychological distress.

\section{Discussion}

This study shows that HRQoL, as reported by an unselected hospital-based cohort of patients with CF, was generally high, except for Social Limitations, Energy/Wellbeing and Treatment Burden. However, almost one quarter of the patients scored positive for psychological distress, and the prevalence increased from children (17\%) to adolescents (27\%) and adults (31\%), although not significantly. Compared to their peers, negative for psychological distress, these patients reported lower levels of HRQoL in most domains and, after adjustment for possible confounders, the differences were statistically significant for Social Limitations, Body Image, and presence of Symptoms. Moreover, we found a strong, statistically significant association between unemployment and presence of psychological distress, independently from demographic, medical and quality of life factors. No effect of gender and clinical variables on psychological distress was found, including a diagnosis based on screening.

The high HRQoL scores reported by CF patients are a common finding in many studies (17). We found that the differences in 
CFQ domain scores were influenced by age. Older patients reported lower median scores in Energy/Wellbeing and Treatment Burden, as a likely effect of the progressive nature of the disease. Treatment constraints were also reported as burdensome by children, reflecting the daily multi-component regimen necessary to control symptoms and prevent complications (1). Compared to their older peers, children's scores were lower for Eating Disturbances and especially perceived Social Limitations. Fear of infection and overprotection by parents may contribute to an explanation of the latter finding (18). Children are less likely than older patients to have bacterial lung colonization, and are therefore more subjected to restriction of social contact and hospital segregation policies aimed at preventing infections (19). However, in our study patients with lower Social Limitations scores were over four times more likely to show psychological distress, even after adjustment for potential confounders. Although we cannot exclude the fact that social problems were the consequence rather than the predictor of psychological distress, the lowest social functioning scores, found in children compared to adolescents, seem to suggest a causative role.

Additional HRQoL measurements, associated with increased likelihood of psychological distress in multivariable analysis, were unsatisfactory Body Image and Symptoms. In contrast, neither the patients' age and sex, nor medical factors, such as FEV1\%, chronic lung infection and Pseudomonas colonization, appeared to exert any effect, indicating that individual subjective perceptions may be stronger predictors of psychological distress than actual disease severity. Similar findings were reported by DiGirolamo and Quittner et al. (20) in a sample of 45 adolescents with CF, where no association between disease severity, age or sex, and reported distress was found.
Several studies have described psychological problems in patients with CF21 but only three, to our knowledge, have explored the relationship with HRQoL (4, 5, 22). Szyndler et al. (22) found that Australian adolescents with CF exhibited psychopathology levels lower than their non-diseased peers (12.5\%); however, increased psychopathology was associated with lower ratings on several quality of life scores. Riekert et al. (4) found a 30\% prevalence of depressive symptoms among adult patients, a figure similar to ours; in all HRQoL domains, patients with both depressive symptoms and poor lung function reported significantly worse scores than those without depressive symptoms, regardless of lung function status. According to Quittner et al. (15) these results support the idea that depression magnifies the effect of chronic illnesses, such as CF and others. Finally Havermans et al. (5) described in a sample of 57 adults with CF the relationship between depressive symptoms and lower scores for Body Image, Emotions, and Eating Disturbances, and between anxiety and several HRQoL domains, including Social Limitations and Respiratory Symptoms. Similarly to the study by Riekert et al. (4), no adjustment other than for FEV1\% was performed.

In this study, we found a strong, statistically significant association between unemployment and the presence of psychological distress, independently from demographic, medical and HRQoL factors. Similar results were reported by Burker et al. (23), who found that FEV1\% did not discriminate between patients who were or were not working, and that workers had lower depression scores. They concluded that either the increased socialization and support linked to a working environment, better income, or distraction from the symptoms of CF may contribute to explain these findings. More recently, Havermans et al. (24) found that adult patients who were neither working nor studying had 
greater disease severity; nevertheless, working or studying patients reported higher HRQoL scores in Role, Physical functioning and Social Limitations, even after controlling for clinical variables.

We found no effect of screening-based CF diagnosis on psychological distress. The association was not significant in multivariable analysis $(\mathrm{p}=0.06)$, possibly because of the small number of screening diagnoses in our population. In Lazio, neonatal CF screening has only been systematically offered since 1993. Thus, our findings pertain mainly to children, and we do not know whether the protective effect will be maintained with growth.

Newborn screening for cystic fibrosis (NBS) is, despite some evidence of its benefits, $(25,26)$ still controversial $(27)$. Anxiety linked to inconclusive or false-positive test results, risk of premature bacterial colonization, due to earlier exposure to the medical environment, and the hazards of carrier detection in a multiethnic society, are a matter of debate (28). The actual benefits of NBS appear less clear-cut than for other types of neonatal screening programs $(26,28)$. The Wisconsin randomized clinical trial showed nutritional benefits, but no long-term effects on pulmonary prognosis (26). Koscik and colleagues (29) reported a link between NBS diagnosis and improved cognitive outcomes, but failed to demonstrate any benefit for HRQoL (7). On the other hand, delayed diagnosis in the face of clinical symptoms is in itself a major source of anxiety for parents, leading to lack of trust in the medical profession and to "doctor shopping" (30). Most parents appear to be supportive of NBS, in the belief that early detection and intervention will improve the health of their children and reduce the stress of a delayed diagnosis $(30,31)$. This positive parental attitude might affect patients' beliefs and the likelihood of developing psychological distress, as suggest- ed by Szyndler et al. (22) who attributed the low levels of psychopathology found in adolescents to the large screening uptake in Australia. Early psycho-social care in a dedicated structure can also be an explanation.

\section{Limitation of study}

This study has limitations. The hospital based group of 115 patients may not be representative of the national or regional population of patients with CF. Although we used standardized validated questionnaires to measure HRQoL and psychological distress, limitations inherent in patient self-report assessment and the choice of cut-off points should be considered. Different instruments were used to measure psychological distress in children and adolescents on the one hand, and adults on the other, and, most noticeably, "cases" were distinguished from "non-cases" using a combination of two different constructs, which can bias the comparison. Further, we did not perform a preliminary crosscultural validation, and no clinical diagnosis of mental illness was available. As previously pointed out, a cross-sectional study cannot identify with certainty the direction of the detected associations, and we cannot state with certainty that lower HRQoL scores and unemployment are the causes, rather than the consequence, of psychological distress. Longitudinal prospective studies are needed to measure the incidence of psychological distress and its time of occurrence. However, we were able to compare the levels of HRQL and psychological distress in a population of children, adolescents and adults, and the high response rate is reassuring in terms of internal validity.

\section{Practical implications}

The findings of this study have implications for the practical management of patients with 
cystic fibrosis, and for screening policies. Our population is characterized by stable clinical conditions and $\mathrm{FEV} 1 \%$ values higher than those commonly reported in the literature. HRQoL scores are consistently high. However, the very low Social Limitations scores found in children are a matter of concern, particularly for the association with psychological distress. Ways should be found to balance protection from infection with the need to preserve opportunities for social contacts and leisure time, avoid isolation and support healthy psychosocial development. HRQoL and psychological problems should play a definite role in assessing the efficacy of any new development of therapy, to introduce its relevant issues in more patient-centred care.

\section{Conclusions}

Systematic screening for psychological distress is useful to identify individuals who may benefit from professional assessment and treatment, and should become part of the routine management of CF patients (32). The use of validated screening questionnaires, such as GHQ-12, and YSR, may facilitate recognition of psychological problems in a non-psychiatric setting. The protective effect of neonatal screening on psychological distress was an unexpected finding, and further studies are needed to confirm it and explore the underlying mechanism. Should the finding be confirmed, however, the case in favour of neonatal CF screening would be stronger.

Acknowledgement: The study was supported by the Italian Ministry of Health, grant n. 200402N001322. There is no conflict of interest to declare.

Authors'contributions: Conception and design: VDC, EM, MC; Acquisition, analysis and interpretation of data: VL, SB, SP; Drafting the article VDC, $\mathrm{MC}$; Revising it critically for important intellectual content: VL.

Conflict of interest: The authors declare that they have no conflict of interest.

\section{References}

1. Cystic Fibrosis Foundation Patient Registry. 2007 Annual Data Report. Bethesda, Maryland. Cystic Fibrosis Foundation 2008.

2. Wilson IB, Cleary PD. Linking clinical variables with health-related quality of life. J Am Med Assoc. 1995;273:59-65.

3. Gee L, Abbott J, Hart A, Conway SP, Etherington C, Webb AK. Associations between clinical variables and quality of life in adults with cystic fibrosis. J Cyst Fibros. 2005;4:59-66.

4. Riekert KA, Bartlett SJ, Boyle MP, Krishnan JA, Rand CS. The association between depression, lung function, and health-related quality of life among adults with cystic fibrosis. Chest. 2007;132:231-7.

5. Havermans T, Colpaert K, Dupont LJ. Quality of life in patients with cystic fibrosis: association with anxiety and depression. J Cyst Fibros. 2008;7:5814.

6. Gee L, Abbott J, Conway SP, Etherington C, Webb AK. Quality of life in cystic fibrosis: the impact of gender, general health perceptions and disease severity. J Cyst Fibros. 2003;2:206-13.

7. Koscik RL, Douglas JA, Zaremba K, Rock MJ, Splaingard ML, Laxova A, et al. Quality of life of children with cystic fibrosis. J Pediatr. 2005;147:S64-S68.

8. Bodnar R, Kadar L, Holics K, Ujhelyi R, Kovacs $\mathrm{L}$, Bolbas K, et al. Factors influencing quality of life and disease severity in Hungarian children and young adults with cystic fibrosis. Ital J Pediatr. 2014; $40: 50-60$.

9. Fuchs HJ, Borowitz DS, Christiansen DH, Morris EM, Nash ML, Ramsey BW, et al. Effect of aerosolized recombinant Human DNase on exacerbations of respiratory symptoms and on pulmonary function in patients with cystic fibrosis. NEJM. 1994;331:637-42.

10. Henry B, Aussage P, Grosskopf C, Goehrs JM. Development of the Cystic Fibrosis Questionnaire (CFQ) for assessing quality of life in pediatric and adult patients. Qual Life Res. 2003;12:63-76.

11. Evaluer la Qualité de vie dans la mucoviscidose: Les questionnaires qualité de vie (CFQ). Manuel d'utilisation. Neuilly-sur-Seine: Produits Roche; 2001.

12. Goldberg DP. The detection of psychiatric illness by questionnaire. London, Oxford University Press, 1972. 
13. Piccinelli M, Bisoffi G, Bon MG, Cunico L, Tansella M. Validity and test-retest reliability of the Italian version of the 12-item General Health Questionnaire in general practice: a comparison between three scoring methods. Compr Psychiatry. 1993;34:198-205.

14. Achenbach TM, Rescorla LA. Manual for the ASEBA school-Age Forms and Profiles: University of Vermont, Research Center for Children, Youth, and Families. Burlington (VT);2001.

15. Quittner AL, Barker DH, Snell C, Grimley ME, Marciel K, Cruz I. Prevalence and impact of depression in cystic fibrosis. Curr Opin Pulm Med. 2008;14:582-8.

16. Cacciari E, Milani S, Balsamo A, Spada E, Bona G, Cavallo L. Italian cross-sectional growth charts for height, weight and BMI (2 to $20 \mathrm{yr}$ ). J Endocrinol Invest. 2006;29(7):581-93.

17. Abbott J. Health-related quality of life measurement in cystic fibrosis: advances and limitations. Chron Respir Dis. 2009;6:31-41.

18. Ullrich G, Wiedau-Gors S, Steinkamp G, Bartig HJ, Schulz W, Freihorst J. Parental fears of pseudomonas infection and measures to prevent its acquisition. J Cyst Fibros. 2002;1:122-30.

19. Cystic Fibrosis Trust 2004. Pseudomonas Aeruginosa in people with cystic fibrosis. Suggestions for prevention and control. Bromley, Kent(enquiries@ cftrust.co.uk): Cystic Fibrosis Trust, 2001.

20. DiGirolamo AM, Quittner AL, Ackerman V, Stevens J. Identification and assessment of ongoing stressors in adolescents with a chronic illness: An application of the Behavior Analytic Model. Journal Clin Child Psychol. 1997;26:53-66.

21. Pfeffer PE, Pfeffer JM, Hodson ME. The psychosocial and psychiatric side of cystic fibrosis in adolescents and adults. J Cyst Fibros. 2003;2:61-8.

22. Szyndler JA, Towns SJ, van Asperen PP, McKay KO. Psychological and quality of life in adolescents with cystic fibrosis. J Cyst Fibros 2005; 4:135-144.
23. Burker EJ, Sedway J, Carone S. Psychological and educational factors: better predictors of work status than FEV1 in adults with cystic fibrosis. Pediatr Pulm. 2004;38:413-18.

24. Havermans T, Colpaert K, Vanharen L, Dupont LJ. Health related quality of life in cystic fibrosis: to work or not to work? J Cyst Fibros. 2009;8:21823.

25. Salvatore D, Buzzetti R, Baldo E, Forneris MP, Lucidi V, Manunza D, et al. An overview of international literature from cystic fibrosis registries 2. Neonatal screening and nutrition/growth. J Cyst Fibros. 2010(2):75-83.

26. Southern KW, Mérelle MME, Dankert-Roelse JE, Nagelkerke A. Newborn screening for cystic fibrosis. Cochrane Database Syst Rev. 2009; (1):CD001402.

27. Rosenthal M. Newborn screening for cystic fibrosis: the motion against. Voices in the wilderness. Paed Resp Rev. 2008;9:295-300.

28. Balfour-Lynn IM. Newborn screening for cystic fibrosis: evidence for benefit. Arch Dis Child. 2008;93:7-10.

29. Koscik RL, Farrell PM, Kosorok MR, Zaremba KM, Laxova A, Lai $\mathrm{H}$, et al. Cognitive function of children with cystic fibrosis: deleterious effect of early malnutrition. Pediatrics 2004;113:1549-58.

30. Tluczek A, Becker T, Laxova A, Grieve A, Racine Gilles CN, Rock MJ, et al. Relationships among health-related quality of life, pulmonary health, and newborn screening for cystic fibrosis. Chest 2011;140:170-77.

31. Kharrazi M, Kharrazi LD. Delayed diagnosis of cystic fibrosis and the family perspective. J Pediatr. 2005;47:S21-S25.

32. Smyth AR, Bell SC, Bojcin S, Bryon M, Duff A, Flume P, et al. European cystic fibrosis $S$ standards of care: best practice guidelines. J Cyst Fibros. 2014; 13:S23-42. 